\title{
Intussusception of Appendiceal Adenoma Mimicking Invasive Carcinoma
}

A 73-year-old man presented with lower abdominal pain. The physical examination revealed a right lower abdominal mass about $6 \mathrm{~cm}$ in diameter, without peritoneal signs. Abdominal ultrasonography and computed tomography revealed a "target sign" in the right lower abdomen, and intussusception was therefore strongly suspected. The right lower abdominal mass was not palpable 1 week later, when colonoscopy was performed. Colonoscopy demonstrated a protruding mass $2 \mathrm{~cm}$ in diameter in the cecum (Figure 1). The orifice of the appendix was not seen. The surface of the tumor was irregular; a depression was present on the top of the tumor, and there was a slight protrusion within the depression. Submucosal

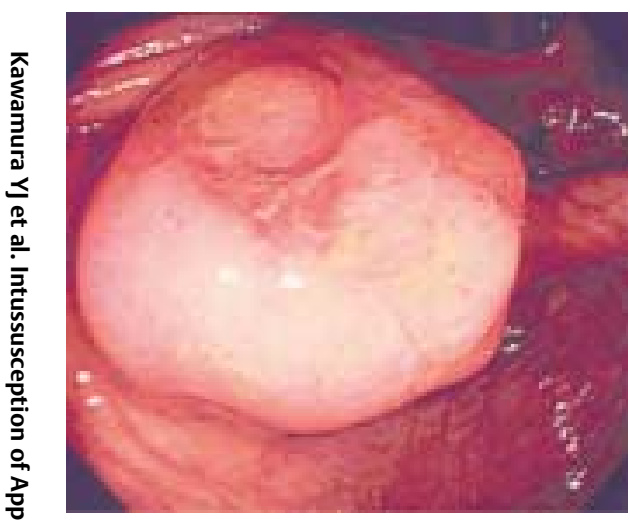

Figure 1 Conventional colonoscopy. The tumor is protruding, and the surface is irregular. Submucosal invasion is suspected

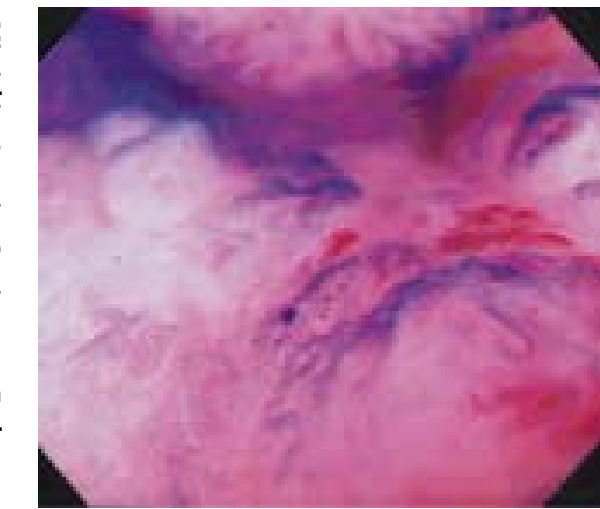

Figure 2 Magnifying colonoscopy using the dye-spray method. The pattern of colonic pits is irregular (type V in Kudo's classification, which corresponds to submucosal invasion) invasion was therefore suspected. Magnifying colonoscopy with dye spraying revealed an irregular pattern of colonic pits (Figure 2), which also suggested submucosal invasion.

Although pathological examination of three biopsy specimens of the tumor revealed adenoma, surgical treatment consisting of ileocecal resection with lymphnode dissection was performed. The postoperative course was uneventful. Pathological examination of the surgical specimen revealed tubulovillous adenoma with moderate atypia, without carcinoma (Figure 3).

Both benign and malignant neoplasms have also been reported to be associated with appendiceal intussusception [1-3]. The differential diagnosis between benign and malignant polyps, and between mucosal and invasive lesions, is particularly important for determining the treatment of choice, since submucosally invasive cancer may be associated with nodal involvement, and extirpation of the affected colon and nodal dissection should there-

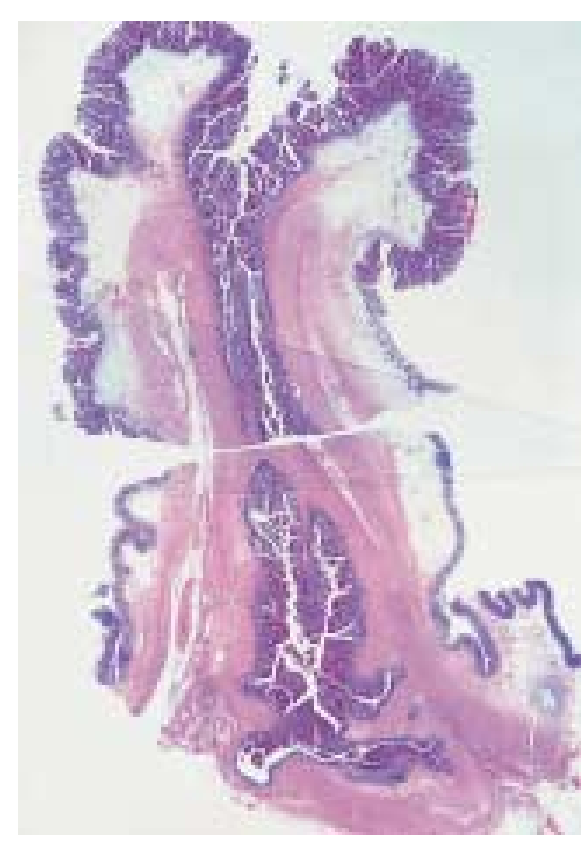

Figure 3 Resected specimen. Pathological examination revealed adenoma with moderate atypia. There were no foci of malignancy fore be considered in such cases [4-5]. However, assessment of the depth of invasion of appendiceal tumors is considered to be very difficult, particularly when intussusception is present. Epithelial tumors in an intussuscepted appendix should therefore be treated with great care. If submucosal invasion cannot be ruled out, we consider that oncological colectomy with lymph-node dissection is the treatment of choice.

\section{Y. J. Kawamura, N. Toyama,}

T. Kasamatsu, M. Ota, F. Konishi

Dept. of Surgery, Jichi Medical School, Omiya Medical Center, Saitama, Japan

\section{References}

${ }^{1}$ Jones CD, Eller DJ, Coates TL. Mucinous cystadenoma of the appendix causing intussusception in an adult. Am J Gastroenterol 1997; 92: 898-899

2 Tonsekar KS, Cacdac R, Ashare R, Libcke $\mathrm{JH}$. Villous adenoma of the vermiform appendix with cecal intussusception: a case report and review of literature. Am Surg 1994; 60: 982 - 984

${ }^{3}$ Chen YC, Chiang JM. Appendiceal intussusception with adenocarcinoma mimicking a cecal polyp. Gastrointest Endosc 2000; 52: 130-131

${ }^{4}$ Morson BC, Busey HJR. Policy of local excision for early cancer of the colorectum. Gut 1977; 18: 1045 - 1050

${ }^{5}$ Kawamura YJ, Sugamata Y, Yoshino K et al. Endoscopic resection for submucosally invasive colorectal cancer: is it feasible? Surg Endosc 1999; 13: 224-227

\section{Corresponding Author}

\section{Y. J. Kawamura, M.D.}

Dept. of Surgery

Jichi Medical School

Omiya Medical Center

1-847 Amanuma-cho Saitama-shi

Saitama 330-8503

Japan

Fax: $\quad$ + 81-48-648-5188

E-mail: kawamura@omiya.jichi.ac.jp 